\title{
@creative
}

ISSN 2590-9770

The Art of Discrete and Applied Mathematics 2 (2019) \#P1.09

https://doi.org/10.26493/2590-9770.1228.eb5

(Also available at http://adam-journal.eu)

\section{Infinite benzenoids}

\author{
Nino Bašić * \\ FAMNIT, University of Primorska, Glagoljaška 8, 6000 Koper, Slovenia \\ IAM, University of Primorska, Muzejski trg 2, 6000 Koper, Slovenia \\ IMFM, Jadranska 19, 1000 Ljubljana, Slovenia
}

Received 6 December 2017, accepted 28 January 2019, published online 30 December 2019

\begin{abstract}
A family of benzenoids, called convex benzenoids, was introduced in 2012 by Cruz, Gutman and Rada. In a later paper by the present author et al., several equivalent characterisations of convex benzenoids have been given and their equivalence was proved. Along the way an infinite benzenoid called the half-plane was used for the purpose of theoretical reasoning. In this short paper, some properties of infinite benzenoids are discussed. It is proved that their boundary consists of countably many connected components. Convex infinite benzenoids are classified and it is proved that there are only countably many convex infinite benzenoids, whilst there are uncountably many infinite (non-convex) benzenoids. We also show that there are countably many infinite benzenoids which have a finite number of $1 \mathrm{~s}$ in their boundary-edges code.
\end{abstract}

Keywords: Infinite benzenoid, hexagonal system, convex benzenoid, boundary-edges code, half-plane, countable set.

Math. Subj. Class.: 05C10, 92E10, 03E75

\section{Introduction}

Benzenoids are important compounds in chemistry and have been extensively studied during the past few decades. For an exhaustive treatment of the topic see the classical reference by Cyvin and Gutman [9]. The reader may want to consult references [5, 6] and [7] for advanced treatments. In the present paper, we study their associated benzenoid graphs from a purely mathematical viewpoint. Hereinafter, the word benzenoid is used as a synonym for benzenoid graph. We build upon theoretical treatment of benzenoids from [2]. The framework of [2] allows for generalisation to structures called infinite benzenoids. In 2012

*The author has been supported in part by ARRS Slovenia (projects P1-0294 and N1-0032). The author would like to thank Tomaž Pisanski for fruitful discussions during the preparation of the manuscript.

E-mail address: nino.basic@famnit.upr.si (Nino Bašić)

(ㄷ)(7) This work is licensed under https://creativecommons.org/licenses/by/4.0/ 
a family of convex benzenoids was introduced by Cruz, Gutman and Rada [4]. In a recent work [3], we presented several alternative characterisations of convex benzenoids. In this work, we study properties of convex infinite benzenoids. We assume that the reader is familiar with results of elementary set theory (e.g., the union of countably many countable sets is countable). For definitions of set-theoretic terms such as countable set and uncountable set, consult a standard reference such as [10] or [12]. This paper further develops several ideas already present in the $\mathrm{PhD}$ thesis of the author [1].

\section{The hexagonal grid}

Let us consider the infinite cubic plane graph $\mathcal{H}$, called the hexagonal grid. (For an introduction to infinite graphs, consult Chapter 8 of [8].) It comprises infinitely many hexagonal faces each of which is incident with 6 edges and 6 vertices. We say that faces $a, b \in F(\mathcal{H})$ are adjacent if they are different and share an edge. The faces of $\mathcal{H}$ will be simply called hexagons. Two hexagons are neighbours if they have exactly one edge in common. Sometimes, it is convenient to introduce a coordinate system on the hexagonal grid $\mathcal{H}$, as shown in Figure 1. Let $h \in \mathcal{H}$. Then $\xi(h)$ and $\eta(h)$ will denote the first and second coordinate

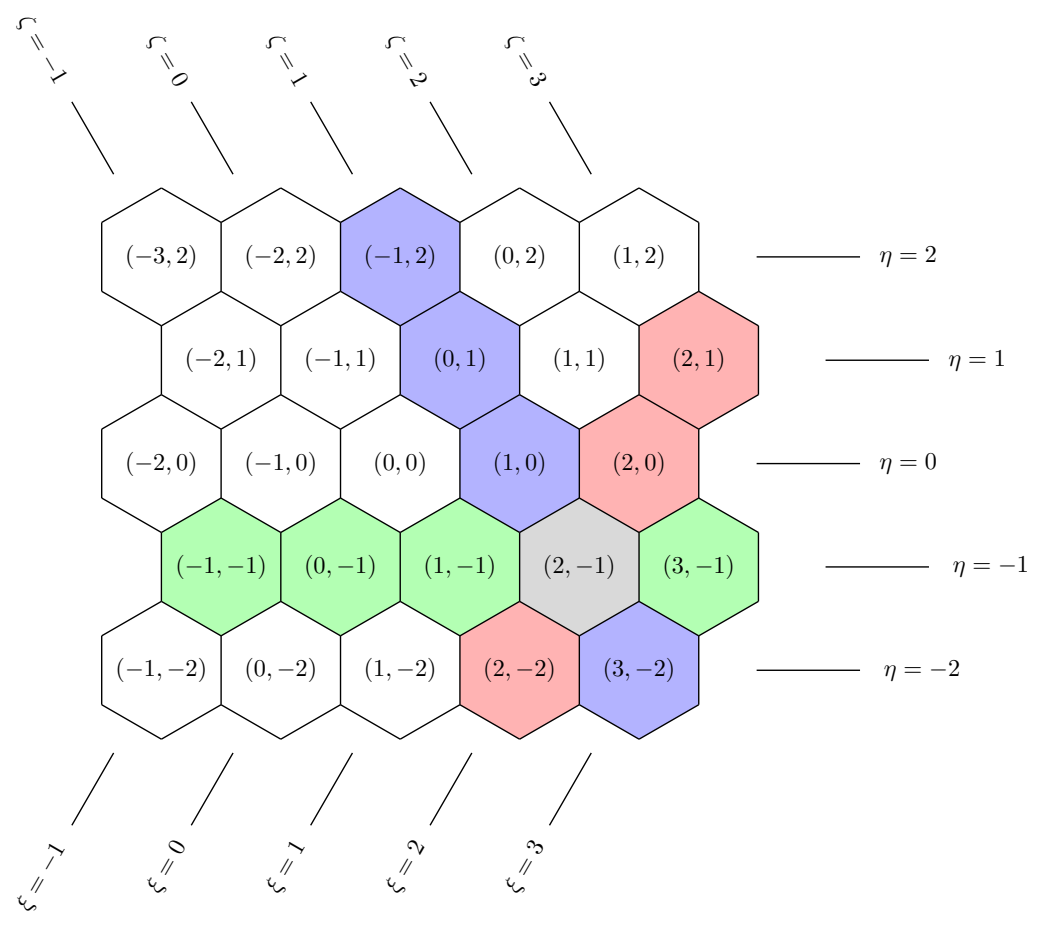

Figure 1: Coordinate system on $\mathcal{H}$.

of $h$ as shown in Figure 1. We may introduce yet another coordinate $\zeta(h)$, but it is not independent of the previous two since $\zeta(h)=\xi(h)+\eta(h)$. For a detailed description of the coordinate system on $\mathcal{H}$, see [1] or [3]. 


\section{Hexagonal systems and benzenoids}

An arbitrary subset of faces $\mathcal{K} \subseteq F(\mathcal{H})$, together with all vertices and edges that are incident with at least one member of $\mathcal{K}$, is called a hexagonal system. We will denote the corresponding hexagonal system simply by $\mathcal{K}$, even though it is formally a subset of faces. Two hexagonal systems are isomorphic if one can be obtained from the other by an isometry of the plane that leaves $\mathcal{H}$ invariant. Hexagons $a \in \mathcal{K}$ and $b \in \mathcal{K}$ belong to the same connected component of the hexagonal system if there exists a sequence of hexagons $h_{1}=a, h_{2}, \ldots, h_{n}=b$ such that $h_{i}$ and $h_{i+1}$ are adjacent for each $i=1, \ldots, n-1$ and $\left\{h_{1}, \ldots, h_{n}\right\} \subseteq \mathcal{K}$. When all hexagons of the sequence are pairwise distinct, it is called a path between $a$ and $b$ in $\mathcal{K}$. The interval between $a$ and $b$ in $\mathcal{K}$, denoted $I_{\mathcal{K}}(a, b)$, is the set of hexagons which are contained on any of the shortest paths between $a$ and $b$. A hexagonal system is connected if it has a single connected component. A subset of faces $\mathcal{K} \subseteq F(\mathcal{H})$ gives rise to two hexagonal systems: $\mathcal{K}$ and its complement $\mathcal{K}^{\complement}$.

We define a benzenoid in the following way:

Definition 3.1. A benzenoid is a connected hexagonal system $\mathcal{K}$, such that each connected component of the complement $\mathcal{K}^{\complement}$ is infinite.

A benzenoid is finite if it has a finite number of hexagons. Otherwise, it is called an infinite benzenoid.

Example 3.2. Let $k \in \mathbb{Z}$. Infinite hexagonal systems

$$
\begin{aligned}
\Xi_{k} & =\{h \in \mathcal{H} \mid \xi(h)=k\}, \\
\mathrm{H}_{k} & =\{h \in \mathcal{H} \mid \eta(h)=k\}, \text { and } \\
\mathrm{Z}_{k} & =\{h \in \mathcal{H} \mid \zeta(h)=k\}
\end{aligned}
$$

will be called lines. They are all benzenoids and they are all isomorphic to each other.

The hexagonal system $\mathcal{K}_{1}$ on Figure 2 is defined as

$$
\mathcal{K}_{1}=\{h \in \mathcal{H} \mid \eta(h) \equiv 0 \quad(\bmod 2)\}=\bigcup_{k \in \mathbb{Z}} \mathrm{H}_{2 k} .
$$

$\mathcal{K}_{1}$ is not a benzenoid, because it is not connected. $\mathcal{K}_{1}$ consists of infinitely many disjoint

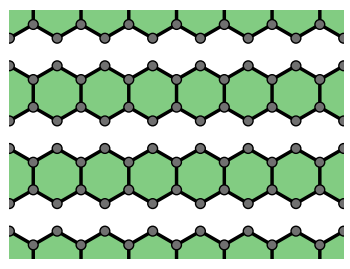

$\mathcal{K}_{1}$

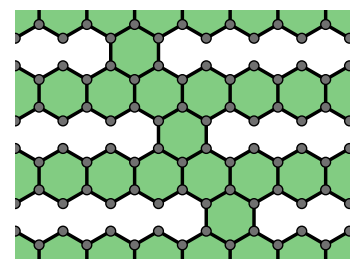

$\mathcal{K}_{2}$

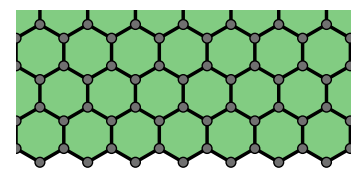

$\mathcal{K}_{3}$

Figure 2: Infinite hexagonal systems $\mathcal{K}_{1}, \mathcal{K}_{2}$ and $\mathcal{K}_{3}$.

lines and is isomorphic to its complement $\mathcal{K}_{1}^{\complement}$.

$\mathcal{K}_{2}$ is obtained from $\mathcal{K}_{1}$ by adding another line (with a different slope), i.e., $\mathcal{K}_{2}=$ $\mathcal{K}_{1} \cup \mathrm{Z}_{0}$. The hexagonal system $\mathcal{K}_{2}$ is a benzenoid. 
Hexagonal system $\mathcal{K}_{3}$ is defined as

$$
\mathcal{K}_{3}=\{h \in \mathcal{H} \mid \eta(h) \geq 0\} .
$$

We call it a convex half-plane. In the present work, we will simply call it a half-plane, since we will not consider other types of (non-convex) half-planes. It is an infinite benzenoid that is isomorphic to its complement $\mathcal{K}_{3}^{\complement}$.

The following theorem tells us that there are as many (non-isomorphic) infinite benzenoids out there as there are real numbers.

Theorem 3.3. There exist uncountably many mutually non-isomorphic infinite benzenoids.

Proof. The interval $[0,1)$ has the cardinality of the continuum. Each number $x \in[0,1)$ can be written in its binary representation

$$
0 . x_{1} x_{2} x_{3} x_{4} \ldots
$$

Note that $x_{i} \in\{0,1\}$ for each $i \in \mathbb{N}$ and that

$$
x=\sum_{i=1}^{\infty} x_{i} \cdot 2^{-i} .
$$

The sequence $\left\{x_{i}\right\}_{i=1}^{\infty}$ is uniquely determined if we require that for each $n \in \mathbb{N}$ there exists an integer $m>n$ such that $x_{m} \neq 1$. For example, the number $\frac{11}{16}$ can be written in binary representation as 0.1011 .

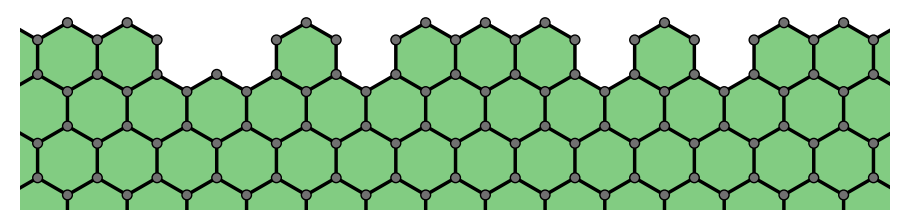

Figure 3: Infinite benzenoid $\mathcal{P}_{\frac{11}{16}}$.

We will assign an infinite benzenoid to every such sequence $\left\{x_{i}\right\}_{i=1}^{\infty}$. Define

$$
\mathcal{P}_{x}=\{h \in \mathcal{H} \mid \eta(h) \leq 0\} \backslash\left(\{(-2,0),(-1,0)\} \cup\left\{(2 i-1,0) \mid i \in \mathbb{N} \wedge x_{i}=1\right\}\right) .
$$

The infinite benzenoid $\mathcal{P}_{x}$ is obtained from the half-plane $\mathcal{P}=\{h \in \mathcal{H} \mid \eta(h) \leq 0\}$ by removing hexagons $(-2,0),(-1,0)$ and all hexagon $(2 i-1,0)$ where $i \in \mathbb{N}$ and $x_{i}=1$. For example, the infinite benzenoid that corresponds to number $\frac{11}{16}$ is shown in Figure 3.

It is not hard to see that $\mathcal{P}_{x} \nsucceq \mathcal{P}_{y}$ if and only if $x \neq y$. We constructed an injective mapping from the set $[0,1)$ to the class of infinite benzenoids. Therefore, the class of all infinite benzenoids is uncountable.

The boundary of a hexagonal system $\mathcal{K}$ consists of all edges and all vertices that are indicent to (at least) one hexagon of $\mathcal{K}$ and (at lest) one hexagon of $\mathcal{K}^{\complement}$. Those vertices and edges are called boundary vertices and boundary edges, respectively.

The boundary of a finite benzenoid is isomorphic to a cycle graph. The boundary of an infinite benzenoid is a disjoint union of one or more infinite paths. The boundary of $\mathcal{K}_{3}$ in Figure 2 is a single infinite path. The boundary of $\mathcal{K}_{2}$ in Figure 2 consists of infinitely many connected components (that are all infinite paths). 
Proposition 3.4. Let $\mathcal{B}$ be an arbitrary benzenoid. The boundary of $\mathcal{B}$ consists of countably many connected components.

Proof. The edges of $\mathcal{H}$ can be labeled with natural numbers in a spiral fashion as shown in Figure 4. Let us define a mapping from the set of connected components of the boundary

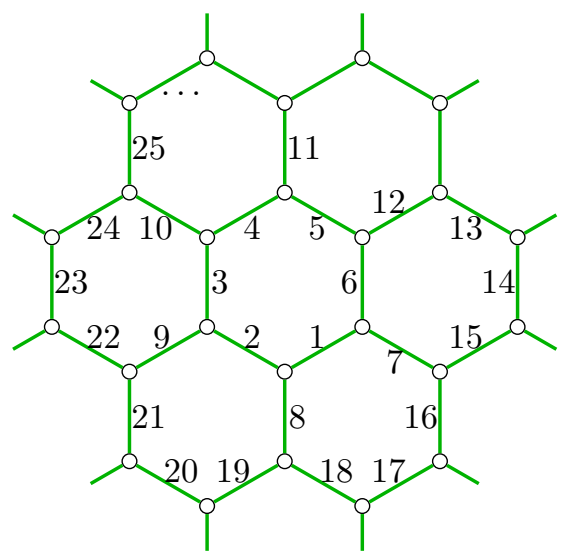

Figure 4: "Spiral" labeling of edges of $\mathcal{H}$.

of $\mathcal{B}$ to natural numbers. Each connected component is mapped to the minimum among all labels of the edges that belong to the component. This mapping to natural numbers is injective and the result follows.

\subsection{The boundary-edges code}

Several combinatorial descriptions of finite benzenoids are readily available. In [11], Hansen et al. presented the boundary-edges code (abbreviated as BEC). The boundary of a finite benzenoid (which is a cycle graph) is described as a cyclic sequence of numbers, each of which counts the number of edges between two consecutive boundary vertices of degree 3. All those numbers are from the set $\{1,2,3,4,5\}$. The only exception here is benzene, which has BEC 6 . In Figure 5, there are several finite benzenoids together with their BECs. The boundary-edges code of $\mathcal{B}$ is denoted $\operatorname{BEC}(\mathcal{B})$.

The code $\operatorname{BEC}\left(\mathcal{B}_{3}\right)=424242$ of $\mathcal{B}_{3}$ from Figure 5 can also be written as $(42)^{3}$, where $s^{k}$ stands for $\underbrace{s s \ldots s}_{k}$.

It is possible to generalise the definition of the BEC to infinite benzenoids. Each connected component of the boundary will be assigned its own BEC. Since a connected component of the boundary of an infinite benzenoid is an infinite path, the BEC can be formally defined as a mapping $\mathbb{Z} \rightarrow\{1,2,3,4,5\}$, i.e. a doubly infinite sequences.

Example 3.5. The boundary of a half-plane has a single connected component with the BEC . . 22222 ..., which can simply be written as $2^{\infty}$.

The boundary of benzenoid $\mathcal{K}_{2}$ in Figure 2 has infinitely many connected components. They all have the same BEC, namely $2^{\infty} 1^{3} 2^{\infty}$.

The boundary of benzenoid $\mathcal{P}_{\frac{11}{16}}$ in Figure 3 has a single connected component with BEC $2^{\infty} 312141^{2} 3231^{2} 41^{2} 32^{\infty}$. 


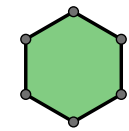

(a) $\mathcal{B}_{1}, \operatorname{BEC}\left(\mathcal{B}_{1}\right)=6$

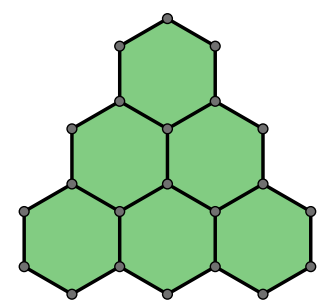

(c) $\mathcal{B}_{3}, \operatorname{BEC}\left(\mathcal{B}_{3}\right)=424242$

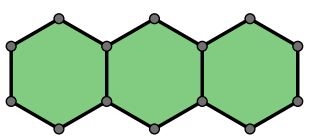

(b) $\mathcal{B}_{2}, \operatorname{BEC}\left(\mathcal{B}_{2}\right)=5252$

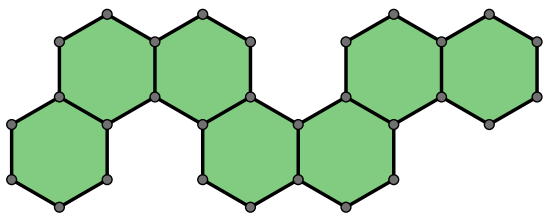

(d) $\mathcal{B}_{4}, \operatorname{BEC}\left(\mathcal{B}_{4}\right)=533113513311$

Figure 5: Examples of finite benzenoids together with their BECs.

Infinite benzenoids can be classified with respect to the number of connected components of the boundary.

Proposition 3.6. Let $n \in \mathbb{N} \cup\{0, \infty\}$. There exists an infinite benzenoid $\mathcal{B}$ with $n$ connected components in the boundary of $\mathcal{B}$. Symbol $\infty$ means that the boundary of $\mathcal{B}$ has (countably) infinitely many connected components.

Proof. The benzenoid $\mathcal{H}$, i.e. the one that contains all hexagons of the infinite hexagonal grid, is the only one with 0 connected components in the boundary. The boundary of halfplane $\mathcal{P}=\{h \in \mathcal{H} \mid \eta(h) \geq 0\}$ has exactly 1 connected component.

Let

$$
\mathcal{B}_{k}=\mathcal{P} \cup \Xi_{0} \cup \Xi_{2} \cup \Xi_{4} \cup \cdots \cup \Xi_{2 k}
$$

It is easy to see that the boundary of $\mathcal{B}_{k}$ has $k+2$ connected components. In other words, for every natural number $n \geq 2$ there exist an infinite benzenoid, namely $\mathcal{B}_{n-2}$, with exactly $n$ connected components in its boundary.

For the case $n=\infty$, take $\mathcal{K}_{2}$ from Figure 2.

\section{Convex (infinite) benzenoids}

Let us recall the metric definition of a convex hexagonal system from [3]:

Definition 4.1. A hexagonal system $\mathcal{K}$ is convex if for any pair of its hexagons $a$ and $b$ the whole interval $I_{\mathcal{H}}(a, b)$ is contained in $\mathcal{K}$.

Theorem 3.3 means that one cannot describe all of the infinite benzenoids algorithmically. This means that there exist infinite benzenoids for which there does not exist a finite computer program for constructing them (even if the program runs infinitely long).

Let us consider convex infinite benzenoids. The whole hexagonal grid $\mathcal{H}$ and the empty set $\emptyset$ are clearly convex. A half-plane is also convex. Each half-plane has a normal which is pointing out of the half-plane (see Figure 6). There are exactly 6 possible directions of 
half-planes. We will denote them as follows:

$$
\begin{aligned}
& \mathcal{H} \mathcal{P}_{\xi}^{+}(n)=\{h \in \mathcal{H} \mid \xi(h) \geq n\}, \\
& \mathcal{H} \mathcal{P}_{\xi}^{-}(n)=\{h \in \mathcal{H} \mid \xi(h) \leq n\}, \\
& \mathcal{H} \mathcal{P}_{\eta}^{+}(n)=\{h \in \mathcal{H} \mid \eta(h) \geq n\}, \\
& \mathcal{H} \mathcal{P}_{\eta}^{-}(n)=\{h \in \mathcal{H} \mid \eta(h) \leq n\}, \\
& \mathcal{H} \mathcal{P}_{\zeta}^{+}(n)=\{h \in \mathcal{H} \mid \zeta(h) \geq n\}, \\
& \mathcal{H} \mathcal{P}_{\zeta}^{-}(n)=\{h \in \mathcal{H} \mid \zeta(h) \leq n\} .
\end{aligned}
$$

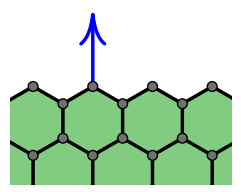

(a) $\mathcal{H} \mathcal{P}_{\eta}^{-}(n)$

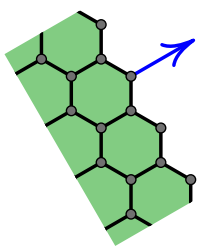

(b) $\mathcal{H} \mathcal{P}_{\zeta}^{-}(n)$

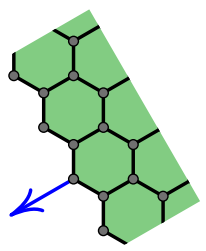

(e) $\mathcal{H} \mathcal{P}_{\zeta}^{+}(n)$

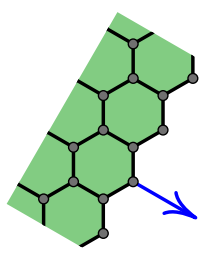

(c) $\mathcal{H} \mathcal{P}_{\xi}^{-}(n)$

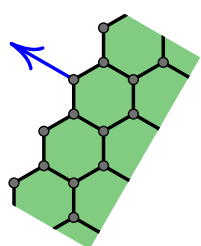

(f) $\mathcal{H} \mathcal{P}_{\xi}^{+}(n)$

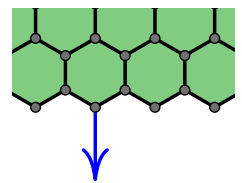

(d) $\mathcal{H} \mathcal{P}_{\eta}^{+}(n)$

Figure 6: The normal of a hexagonal half-plane can point in 6 different directions.

Note that the intersection of two half-planes with the same normal is equal to one of the two. In other words, one is a sub-benzenoid of the other. For example,

$$
\mathcal{H} \mathcal{P}_{\xi}^{+}(3) \cap \mathcal{H} \mathcal{P}_{\xi}^{+}(5)=\mathcal{H} \mathcal{P}_{\xi}^{+}(5)
$$

Therefore, the interection of any number of half-planes is equal to an intersection of at most 6 of those half-planes. For each direction of the normal that is present in the list, we select the half-plane that is contained in all other half-planes that have the same direction. Also, note that all half-planes (4.1) - (4.6) are isomorphic to each other.

Definition 4.2. Let $\mathcal{B}$ be a benzenoid (finite or infinite). The smallest convex benzenoid containing $\mathcal{B}$ is called the convex closure of $\mathcal{B}$ and is denoted $\operatorname{Conv}(\mathcal{B})$.

In [3], the following proposition was proved.

Proposition 4.3. Any intersection of convex (finite or infinite) benzenoids is a convex benzenoid. 
Note that the empty set $\emptyset$ can also be considered as a convex benzenoid. To a chemist, this means nothing concrete. To a mathematician, it is clear that every two members of an empty set satisfy the condition in Definition 4.1, i.e., this condition is void.

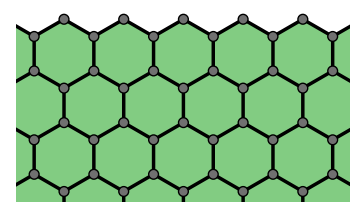

(a) half-plane, $\mathcal{H P}$

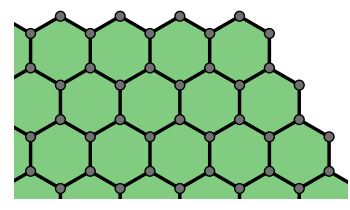

(b) anvil, $\mathcal{A N}$

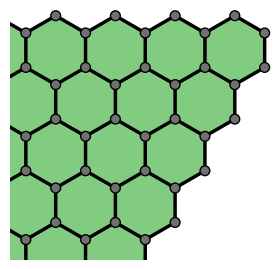

(c) wedge, $\mathcal{W E}$

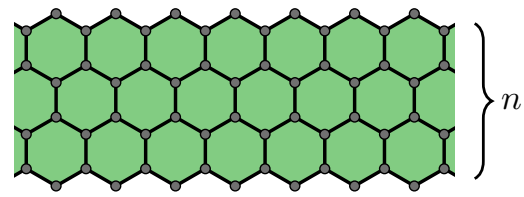

(d) $\operatorname{strip}, \mathcal{S T}(n)$

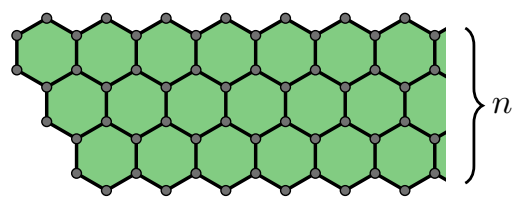

(f) knife, $\mathcal{K N}(n)$

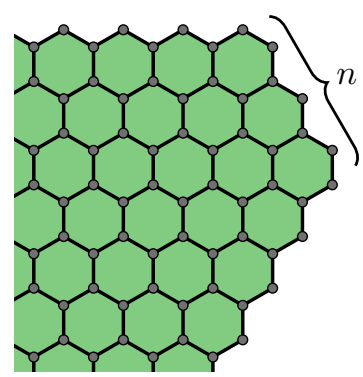

(e) chomped wedge, $\mathcal{C W}(n)$

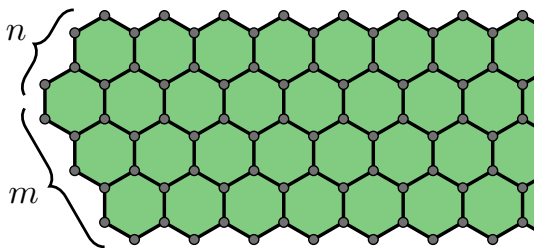

(g) sword, $\mathcal{S} \mathcal{W}(n, m)$

Figure 7: Families of convex infinite benzenoids.

In addition to the hexagonal grid $\mathcal{H}$, the empty set $\emptyset$ and half-plane $\mathcal{H P}$, we introduce the following families of infinite benzenoids (see Figure 7):

(a) The benzenoid $\mathcal{A N}$ in Figure 7(b) is called anvil and is uniquely determined (up to isomorphism) by $\operatorname{BEC}(\mathcal{A N})=2^{\infty} 32^{\infty}$.

(b) The benzenoid $\mathcal{W E}$ in Figure 7(c) is called wedge and is uniquely determined (up to isomorphism) by $\mathrm{BEC}(\mathcal{W E})=2^{\infty} 42^{\infty}$.

(c) A member of the one-parametric family of benzenoids $\mathcal{S T}(n), n \geq 1$, in Figure 7(d) is called a strip (of width $n$ ) and cannot be uniquely determined by the BEC. However, $\mathcal{S} \mathcal{T}(n) \cong \mathcal{H} \mathcal{P}_{\xi}^{+}(0) \cap \mathcal{H} \mathcal{P}_{\xi}^{-}(n)$. Note that $\mathcal{S T}(1)$ is called a line.

(d) A member of the one-parametric family of benzenoids $\mathcal{C W}(n), n \geq 2$, in Figure 7(e) is called a chomped wedge and is uniquely determined (up to isomorphism) by $\operatorname{BEC}(\mathcal{C W}(n))=2^{\infty} 32^{n-2} 32^{\infty}$. 
(e) A member of the one-parametric family of benzenoids $\mathcal{K N}(n), n \geq 1$, in Figure 7(f) is called a knife (of width $n$ ) and is uniquely determined (up to isomorphism) by

$$
\operatorname{BEC}(\mathcal{K} \mathcal{N}(n))= \begin{cases}2^{\infty} 32^{n-2} 42^{\infty} & \text { if } n \geq 2 \\ 2^{\infty} 52^{\infty} & \text { if } n=1\end{cases}
$$

The benzenoid $\mathcal{K} \mathcal{N}(1)$ will also be called a needle.

(f) A member of the two-parametric family of benzenoids $\mathcal{S W}(n, m), m \geq n \geq 2$, in Figure $7(\mathrm{~g})$ is called a sword and is uniquely determined (up to isomorphism) by $\operatorname{BEC}(\mathcal{S W}(n))=2^{\infty} 32^{n-2} 32^{m-2} 32^{\infty}$.

All benzenoids on the above list can be obtaned as intersections of half-planes and are therefore convex by Proposition 4.3.

In [3], the following proposition was proved.

Proposition 4.4. A finite benzenoid $\mathcal{B}$ is convex if and only if it can be obtained as an intersection of half-planes, i.e., if there exist integers $n_{\xi}^{+}, n_{\xi}^{-}, n_{\eta}^{+}, n_{\eta}^{-}, n_{\zeta}^{+}$and $n_{\zeta}^{-}$, such that

$$
\mathcal{B}=\mathcal{H} \mathcal{P}_{\xi}^{+}\left(n_{\xi}^{+}\right) \cap \mathcal{H} \mathcal{P}_{\xi}^{-}\left(n_{\xi}^{-}\right) \cap \mathcal{H} \mathcal{P}_{\eta}^{+}\left(n_{\eta}^{+}\right) \cap \mathcal{H} \mathcal{P}_{\eta}^{-}\left(n_{\eta}^{-}\right) \cap \mathcal{H} \mathcal{P}_{\zeta}^{+}\left(n_{\zeta}^{+}\right) \cap \mathcal{H} \mathcal{P}_{\zeta}^{-}\left(n_{\zeta}^{-}\right)
$$

Let $\mathcal{B}$ be a benzenoid. We define

$$
\eta^{+}=\max _{h \in \mathcal{B}} \eta(h) \quad \text { and } \quad \eta^{-}=\min _{h \in \mathcal{B}} \eta(h) .
$$

If the set $\{\eta(h) \mid h \in \mathcal{B}\}$ is not bounded from above, we write $\eta^{+}=\infty$. Similarly, if the set $\{\eta(h) \mid h \in \mathcal{B}\}$ is not bounded from below, we write $\eta^{-}=-\infty$. Analogously, we define

$$
\xi^{+}=\max _{h \in \mathcal{B}} \xi(h), \quad \xi^{-}=\min _{h \in \mathcal{B}} \xi(h), \quad \zeta^{+}=\max _{h \in \mathcal{B}} \zeta(h) \quad \text { and } \quad \zeta^{-}=\min _{h \in \mathcal{B}} \zeta(h) .
$$

Lemma 4.5. Let $\mathcal{B}$ be a convex benzenoid. Then the following statements hold:

(1) If $\eta^{+}<\infty$ and $\xi^{+}<\infty$ then $\zeta^{+}<\infty$.

(2) If $\eta^{-}>-\infty$ and $\xi^{-}>-\infty$ then $\zeta^{-}>-\infty$.

(3) If $\xi^{+}<\infty$ and $\zeta^{-}>-\infty$ then $\eta^{-}>-\infty$.

(4) If $\xi^{-}>-\infty$ and $\zeta^{+}<\infty$ then $\eta^{+}<\infty$.

(5) If $\eta^{+}<\infty$ and $\zeta^{-}>-\infty$ then $\xi^{-}>-\infty$.

(6) If $\eta^{-}>-\infty$ and $\zeta^{+}<\infty$ then $\xi^{+}<\infty$.

Proof. It is enough to prove statement (1) of the lemma. Other statements will results from the fact that we may use a rotational symmetry of the hexagonal grid $\mathcal{H}$.

Suppose that $\eta^{+}<\infty$ and $\xi^{+}<\infty$. This implies $\mathcal{B} \subseteq \mathcal{H} \mathcal{P}_{\eta}^{-}\left(\eta^{+}\right)$and $\mathcal{B} \subseteq \mathcal{H} \mathcal{P}_{\xi}^{-}\left(\xi^{+}\right)$. Moreover, $\mathcal{B} \subseteq \mathcal{H} \mathcal{P}_{\eta}^{-}\left(\eta^{+}\right) \cap \mathcal{H} \mathcal{P}_{\xi}^{-}\left(\xi^{+}\right)$. But $\mathcal{H} \mathcal{P}_{\eta}^{-}\left(\eta^{+}\right) \cap \mathcal{H} \mathcal{P}_{\xi}^{-}\left(\xi^{+}\right)$is a wedge in which hexagon $\left(\xi^{+}, \eta^{+}\right)$obtains the maximal value of $\zeta$ coordinate. Therefore, $\zeta^{+}<\infty$. 
The next result generalises Proposition 4.4 to infinite benzenoids.

Proposition 4.6. An benzenoid $\mathcal{B}$ (finite or infinite) is convex if and only if it can be obtained as an intersection of half-planes.

Proof. The proof technique used here is similar to the one used in [3] to prove Proposition 4.4. If a benzenoid $\mathcal{B}$ is obtained as an intersection of half-planes then it is convex by Proposition 4.3 .

Now let $\mathcal{B}$ be a convex benzenoid. There are several cases to consider, depending on the values of $\xi^{+}, \xi^{-}, \eta^{+}, \eta^{-}, \zeta^{+}$and $\zeta^{-}$.

Suppose that $\xi^{-}>-\infty, \eta^{-}>-\infty$ and $\zeta^{+}<\infty$. Statements (2), (4) and (6) of Lemma 4.5 imply that $\zeta^{-}>-\infty, \eta^{+}<\infty$ and $\xi^{+}<\infty$, respectively. This means that $\mathcal{B}$ is finite and the result follows from Proposition 4.4.

We need to consider several more cases:

(i) $\xi^{-}=-\infty, \eta^{-}>-\infty$ and $\zeta^{+}<\infty$;

(ii) $\xi^{-}=-\infty, \eta^{-}=-\infty$ and $\zeta^{+}<\infty$;

(iii) $\xi^{-}=-\infty, \eta^{-}=-\infty$ and $\zeta^{+}=\infty$.

Note that because of the symmetry, there are only 3 cases to consider and not $2^{3}$. We provide the proof of case (i). The proofs of (ii) and (iii) are very similar and use the same type of arguments.

(i): Suppose that $\xi^{-}=-\infty, \eta^{-}>-\infty$ and $\zeta^{+}<\infty$. Statements (6) of Lemma 4.5 implies that $\xi^{+}<\infty$. Now consider $\zeta^{-}$and $\eta^{+}$. If $\zeta^{-}>-\infty$ and $\eta^{+}<\infty$ then by statement (5) of Lemma 4.5 we obtain $\xi^{-}>-\infty$, a contradiction. We have two subcases:

$$
\begin{aligned}
& \zeta^{-}=-\infty \text { and } \eta^{+}=\infty \\
& \zeta^{-}=-\infty \text { and } \eta^{+}<\infty
\end{aligned}
$$

Again, by the symmetry argument, we do not need to consider the case $\zeta^{-}>-\infty$ and $\eta^{+}=\infty$. We will now consider case (i.1). The case (i.2) is analogous.

(i.1): From $\eta^{-}>-\infty$ it follows that there exists a hexagon $h_{\eta^{-}} \in \mathcal{B}$ such that $\eta\left(h_{\eta^{-}}\right)=\eta^{-}$. From $\zeta^{+}<\infty$ it follows that there exists a hexagon $h_{\zeta^{+}} \in \mathcal{B}$ such that $\zeta\left(h_{\zeta^{+}}\right)=\zeta^{+}$. And from $\xi^{+}<\infty$ it follows that there exists a hexagon $h_{\xi^{+}} \in \mathcal{B}$ such that $\xi\left(h_{\xi^{+}}\right)=\xi^{+}$. See Figure 8 for an illustration. Let $h_{1}, h_{2} \in \mathcal{H}$ such that $\eta\left(h_{1}\right)=\eta^{-}, \xi\left(h_{1}\right)=\xi^{+}, \zeta\left(h_{2}\right)=\zeta^{+}$and $\xi\left(h_{2}\right)=\xi^{+}$. Because $h_{1} \in I_{\mathcal{H}}\left(h_{\eta-}, h_{\xi^{+}}\right)$and $h_{2} \in I_{\mathcal{H}}\left(h_{\xi^{+}}, h_{\zeta^{+}}\right)$, it follows that $h_{1}, h_{2} \in \mathcal{B}$.

Let $n$ be an arbitrary large integer. From $\zeta^{-}=-\infty$ it follow that there exists a hexagon $h^{\prime} \in \mathcal{B}$ such that $\zeta\left(h^{\prime}\right)=-n$. Let $h^{\prime \prime} \in \mathcal{H}$ be the hexagon with $\zeta\left(h^{\prime \prime}\right)=-n$ and $\eta\left(h^{\prime \prime}\right)=\eta^{-}$. Since $h^{\prime \prime} \in I_{\mathcal{H}}\left(h^{\prime}, h_{1}\right)$ it follow that $h^{\prime \prime} \in \mathcal{B}$. This means that $\mathcal{B}$ contains the needle $\left\{h \in \mathcal{H} \mid \eta(h)=\eta^{-} \wedge \xi(h) \leq \xi^{+}\right\}$. Similarly, $\eta^{+}=\infty$ implies that $\mathcal{B}$ also contains the needle $\left\{h \in \mathcal{H} \mid \zeta(h)=\zeta^{+} \wedge \xi(h) \leq \xi^{+}\right\}$. The line segment between $h_{1}$ and $h_{2}$ is also contained in $\mathcal{B}$.

This means that all boundary hexagons of $\mathcal{H} \mathcal{P}_{\eta}^{+}\left(\eta^{-}\right) \cap \mathcal{H} \mathcal{P}_{\xi}^{-}\left(\xi^{+}\right) \cap \mathcal{H} \mathcal{P}_{\zeta}^{-}\left(\zeta^{+}\right)$are contained in $\mathcal{B}$. But $\mathcal{B} \subseteq \mathcal{H} \mathcal{P}_{\eta}^{+}\left(\eta^{-}\right) \cap \mathcal{H} \mathcal{P}_{\xi}^{-}\left(\xi^{+}\right) \cap \mathcal{H} \mathcal{P}_{\zeta}^{-}\left(\zeta^{+}\right)$and therefore

$$
\mathcal{B}=\mathcal{H} \mathcal{P}_{\eta}^{+}\left(\eta^{-}\right) \cap \mathcal{H} \mathcal{P}_{\xi}^{-}\left(\xi^{+}\right) \cap \mathcal{H} \mathcal{P}_{\zeta}^{-}\left(\zeta^{+}\right)
$$

The remaining cases can be proved using the same approach. 


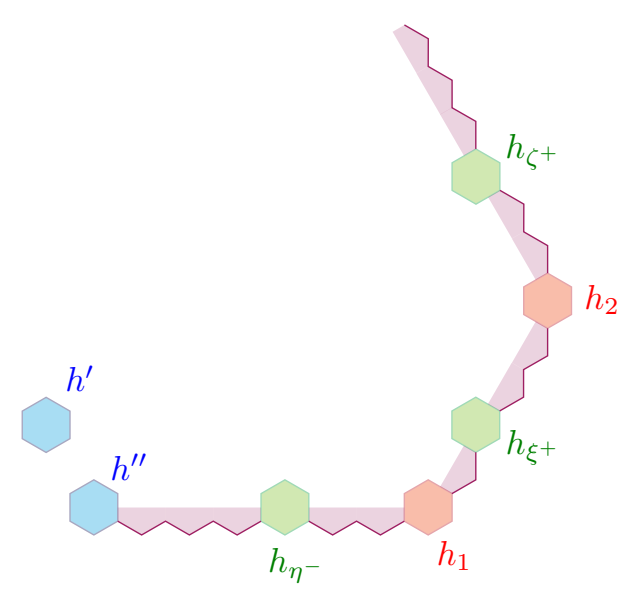

Figure 8: The case (i.1).

We are now able to prove the following theorem.

Theorem 4.7. An infinite benzenoid is convex if and only if it is isomorphic to one of the following:

(a) the hexagonal grid $\mathcal{H}$,

(b) the half-plane $\mathcal{H} \mathcal{P}$,

(c) the anvil $\mathcal{A N}$,

(d) the wedge $\mathcal{W E}$,

(e) a strip $\mathcal{S T}(n)$ for some $n \geq 1$,

(f) a chomped wedge $\mathcal{C W}(n)$ for some $n \geq 2$,

(g) a knife $\mathcal{K N}(n)$ for some $n \geq 1$,

(h) a sword $\mathcal{S W}(n, m)$ for some $m \geq n \geq 2$.

Moreover, all benzenoids from the above list are pairwise non-isomorphic.

Proof. We already know that all infinite benzenoids listed in the statement of the theorem are convex. It is not hard to see that they are also pairwise non-isomorphic.

Suppose that $\mathcal{B}$ is a convex infinite benzenoid. From Proposition 4.6 it follows that it can be obtained as an intersection of half-planes. We can analyse (case by case) all possibilities for the intersection of (any subset) of the 6 half-planes. In this analysis, we either obtain a finite convex benzenoid (they were classified in [3]), which contradicts the assumption that $\mathcal{B}$ is infinite, or one of the above.

Let $\mathcal{F O}$ be the class of all such infinite benzenoids $\mathcal{B}$ with the property that the total number of $1 \mathrm{~s}$ in BECs of all connected components of the boundary of $\mathcal{B}$ is finite. 
Theorem 4.8. There are countably many benzenoids in the class $\mathcal{F O}$.

Proof. Let $\mathcal{O}_{n}, n \geq 0$, be the finite benzenoid defined by $\operatorname{BEC}\left(\mathcal{O}_{n}\right)=\left(32^{n}\right)^{6}$.

Let $\mathcal{B}$ be any benzenoid of the class $\mathcal{F O}$ (see Figure 9 for an example). Because there

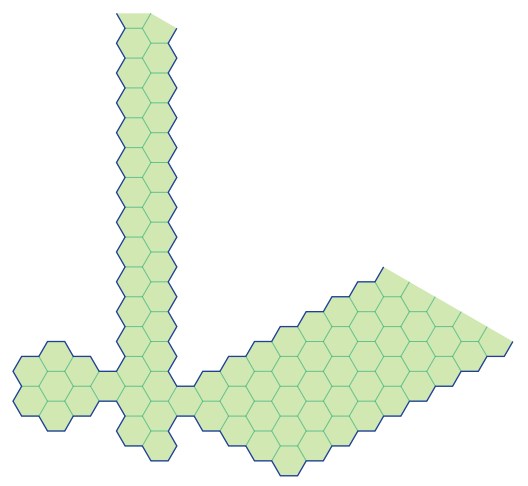

Figure 9: A benzenoid $\mathcal{B} \in \mathcal{F O}$.

are finitely many $1 \mathrm{~s}$ in BECs of $\mathcal{B}$, there exists some $\mathcal{O}^{\prime}=\mathcal{O}_{n}$ for $n$ large enough, such that $\mathcal{O}^{\prime}$ contains all edges that correspond to 1 s in BECs of $\mathcal{B}$ as internal edges (see Figure 10 (a)). Then $\mathcal{B} \backslash \mathcal{O}^{\prime}$ is a hexagonal system that comprises finitely many finite benzenoids and finitely many infinite benzenoids. (In the example in Figure 10(a), there is 1 finite benzenoid and 2 infinite benzenoids.)

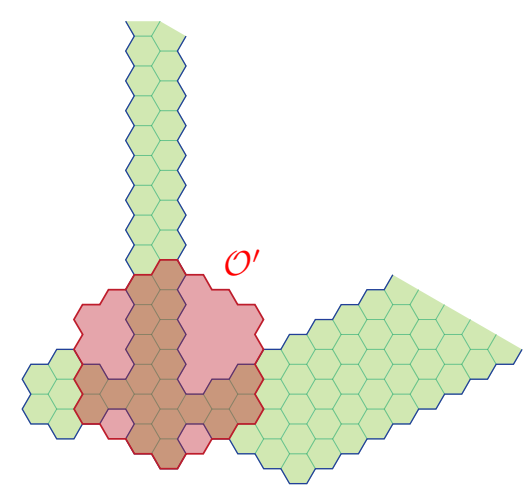

(a)

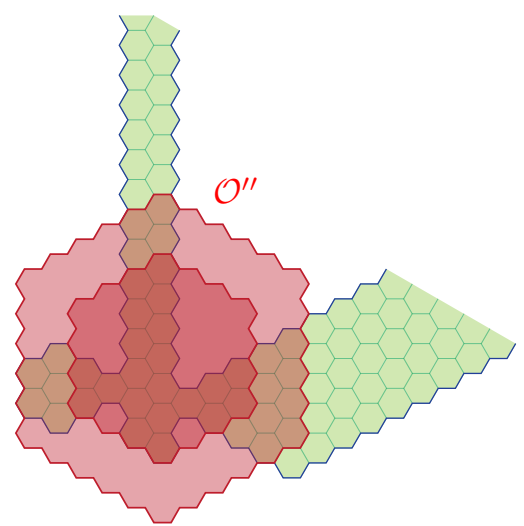

(b)

Figure 10: A benzenoid $\mathcal{B} \in \mathcal{F} \mathcal{O}$ with $\mathcal{O}^{\prime}$ and $\mathcal{O}^{\prime \prime}$.

We can choose a (possibly larger) number $m \geq n$, such that $\mathcal{O}^{\prime \prime}=\mathcal{O}_{m}$ contains $\mathcal{O}^{\prime}$ and all finite connected components of $\mathcal{B} \backslash \mathcal{O}^{\prime}$ (see Figure 10(b)). If we remove all edges of $\mathcal{O}^{\prime \prime}$ from the boundary of $\mathcal{B}$, we obtain an even number of semi-infinite paths. No edge on those semi-infinite paths is an edge that corresponds to a 1 s in BECs of $\mathcal{B}$, because such 
edges are all contained in $\mathcal{O}^{\prime}$. The subsequence of a BEC that corresponds to such a semiinfinite path contains infinitely many $2 \mathrm{~s}$, but only a finite number of $3 \mathrm{~s}, 4 \mathrm{~s}$ and $5 \mathrm{~s}$. Those numbers cause bends in the boundary and too many bends would result in a collision of the boundary (see Figure 11), which is impossible. To encode the benzenoid $\mathcal{B}$, we need the

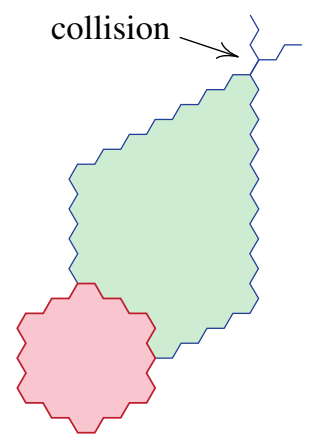

Figure 11: Collision of the boundary of $\mathcal{B}$.

following information:

(i) the number $m$, which determines the $\mathcal{O}^{\prime \prime}$ (we have countably many choices),

(ii) the subset of hexagons of $\mathcal{O}^{\prime \prime}$ that determine $\mathcal{B} \cap \mathcal{O}^{\prime \prime}$ (there are finitely many such subsets),

(iii) vetices on the boundary of $\mathcal{O}^{\prime \prime}$ that are starting vertices of semi-infinite paths (finitely many choices),

(iv) positions and types of bends on each semi-infinite path (there are finitely many bends and each type and position can be encoded by a natural number).

From the above encoding of $\mathcal{B}$ it follows that there are countably many different benzenoids in the class $\mathcal{F} \mathcal{O}$.

Corollary 4.9. There exist countably many mutually non-isomorphic convex infinite benzenoids.

Proof. By Theorem 4.7, a convex infinite benzenoid is isomorphic to one of those listed in the statement of Theorem 4.7. None of them has a 1 in the boundary-edges code of any connected component of the boundary. Therefore, the class of convex infinite benzenoids is a subclass of $\mathcal{F O}$ and the results follows by Theorem 4.8 .

\section{References}

[1] N. Bašić, Algebraic Approach to Several Families of Chemical Graphs, Ph.D. thesis, University of Ljubljana, 2016.

[2] N. Bašić, P. W. Fowler and T. Pisanski, Coronoids, patches and generalised altans, J. Math. Chem. 54 (2016), 977-1009, doi:10.1007/s10910-016-0599-6. 
[3] N. Bašić, P. W. Fowler and T. Pisanski, Stratified enumeration of convex benzenoids, MATCH Commun. Math. Comput. Chem. 80 (2018), 153-172, http: / /match.pmf.kg.ac.rs/ electronic_versions/Match80/n1/match80n1_153-172.pdf.

[4] R. Cruz, I. Gutman and J. Rada, Convex hexagonal systems and their topological indices, MATCH Commun. Math. Comput. Chem. 68 (2012), 97-108, http: / /www • pmf . kg • ac . rs/match/electronic_versions/match68/n1/match68n1_97-108.pdf.

[5] S. J. Cyvin, J. Brunvoll and B. N. Cyvin, Theory of Coronoid Hydrocarbons, volume 54 of Lecture Notes in Chemistry, Springer-Verlag, Berlin, 1991, doi:10.1007/978-3-642-51110-3.

[6] S. J. Cyvin, J. Brunvoll, B. N. Cyvin, R. S. Chen and F. J. Zhang, Theory of Coronoid Hydrocarbons II, volume 62 of Lecture Notes in Chemistry, Springer-Verlag, Berlin, 1994, doi: 10.1007/978-3-642-50157-9.

[7] S. J. Cyvin and I. Gutman, Kekulé Structures in Benzenoid Hydrocarbons, volume 46 of Lecture Notes in Chemistry, Springer-Verlag, Berlin, 1988, doi:10.1007/978-3-662-00892-8.

[8] R. Diestel, Graph Theory, volume 173 of Graduate Texts in Mathematics, Springer, Berlin, 5th edition, 2017, doi:10.1007/978-3-662-53622-3.

[9] I. Gutman and S. J. Cyvin, Introduction to the Theory of Benzenoid Hydrocarbons, SpringerVerlag, 1989, doi:10.1007/978-3-642-87143-6.

[10] P. R. Halmos, Naive Set Theory, Undergraduate Texts in Mathematics, Springer-Verlag, New York, 1st edition, 1974, doi:10.1007/978-1-4757-1645-0.

[11] P. Hansen, C. Lebatteux and M. Zheng, The boundary-edges code for polyhexes, J. Mol. Struct. (THEOCHEM) 363 (1996), 237-247, doi:10.1016/0166-1280(95)04139-7.

[12] T. Jech, Set Theory, Springer Monographs in Mathematics, Springer-Verlag, Berlin, 2003, doi: 10.1007/3-540-44761-x. 